\title{
Needle Placement: A Guide to Predictable Sensory and Motor Responses Based on Variations in Needle Placement During Sacral Neuromodulation Procedures
}

\author{
Melanie Crites-Bachert ${ }^{1}$, Casey Clark ${ }^{2}$ \\ ${ }^{1} 360$ Pelvic Health Institute, A Division of The Center for Men's and Women's Urology Gresham, OR, USA \\ ${ }^{2} \mathrm{C} \&$ S Surgical, Camas, WA, USA
}

Purpose: Providing practitioners with an adjunctive guide that will aid implanters in the ability to predict and, immediately recognize, what suboptimal needle placement looks like (based on visualization of needle/lead placement and the patient's sensory and motor responses), allow cultivation of a more complete and comprehensive level of understanding of the sacral neuromodulation procedure as a whole, and inversely provide a guide for what true optimal needle/lead placement should currently demonstrate (based on current International Continence Society guidelines).

Methods: More than 400 patients underwent sacral neuromodulation procedures from 2011-2018 by a practitioner who is in the top 5\% of implanting physicians in the United States. Common stimulation patterns with motor and sensory responses were observed in patients with suboptimal needle placement intraoperatively.

Results: Reproducible stimulation patterns were observed with common suboptimal needle placement intraoperatively. This allowed the implanting practitioner to immediately identify and correct the needle placement intraoperatively to achieve optimal needle placement and optimal motor and sensory responses for the patients.

Conclusions: By considering the 3-dimensional spatial trajectory of the $\mathrm{S} 3$ nerve, and following this presented guide, the most optimal lead placement with consistently reproducible outcomes that include S3 motor and sensory response on all 4 leads at less than, or equal to, 2 volts can be achieved and can potentially maximize the life of the device while potentially affording patients a more successful outcome.

Keywords: Sacral neuromodulation; Nonobstructive urinary retention; Urinary bladder, overactive; Fecal incontinence; Urinary frequency; Urinary urgency

- Research Ethics: After inquiry with the Institutional Review Board, approval was not required from the board for the reporting of these aggregate pattern observations.

- Conflict of Interest: MCB was a proctor for Medtronic from 2015-2017 and is currently a proctor for Laborie since 2018. CC was a Medtronic employee from 2012-2017 and then resumed employment with Medtronic in late 2018. CC was not an employee of Medtronic during the drafting of this manuscript.

\section{INTRODUCTION}

Sacral neuromodulation (SNM) became a commercially ap- proved therapy in the United States for treating functional disorders of the bladder in 1997 and fecal incontinence in 2011. Since that time, practitioners have had wide-ranging variations
Corresponding author: Melanie Crites-Bachert (iD) https://orcid.org/0000-0003-3066-4548 360 Pelvic Health Institute: A Division of The Center for Men's and Women's Urology, 24076 SE Stark St., Ste. 310, Gresham, OR 97030, USA

E-mail: Mcrites-bachert@1uro.com / Tel: +1-503-492-6510 / Fax: +1-503-492-6502

Submitted: May 14, 2019 / Accepted after revision: October 1, 2019
This is an Open Access article distributed under the terms of the Creative Commons Attribution Non-Commercial License (http://creativecommons.org/licenses/by-nc/4.0/) which permits unrestricted non-commercial use, distribution, and reproduction in any medium, provided the original work is properly cited. 
in outcomes with some patients experiencing a significant reduction in their symptoms, while other patients experience therapy fails. Just recently, the International Continence Society issued a best practice statement for use of SNM citing that there are a variety of issues associated with the use of SNM and obtaining optimal response to the therapy [1]. The large variability in outcomes has been thought, by many, to be partially secondary to inadequate electrode placement that results in suboptimal coupling of the electrode and nerve. That thought prompted many practitioners to address the problem of inadequate electrode placement by finding better and more standardized methods and techniques of placement. While standardizing electrode placement is paramount to creating better understanding of how to minimize the variability in successful outcomes, there remains the need to better understand and recognize what all variations of inadequate electrode placements look like to better augment the standardized placement technique and, adjunctively, further minimize the vast variabilities in successful outcomes.

\section{MATERIALS AND METHODS}

More than 400 SNM procedures including percutaneous nerve evaluation, stage I, full implants, and revisions of lead were performed by a single surgeon from August 2011 to December 2018 on patients ranging from 23-92 years of age including 13 males and 267 females. All patients had been diagnosed with refractory frequency/urgency, nonobstructive urinary retention, or fecal incontinence. The surgeon has been an active implanter of SNM since 2005 and routinely has motor and sensory responses on all 4 lead contact points at $\leq 1 \mathrm{~V}$. The implanting physician for this study is a high-volume implanting physician in the top $5 \%$ of physicians who perform the SNM procedure in the United States. Fluoroscopic renderings of inadequate or "suboptimal" electrode placements (with associated sensory and motor responses) were developed from the aggregate information derived from those procedures to provide a standardized guideline of how to avoid placing the electrode, to further minimize the variability in successful outcomes. After inquiry with the Institutional Review Board, approval was not required from the board for the reporting of these aggregate pattern observations.

Each patient was placed in the prone position with bolsters underneath their lower abdomen and feet. The intention is to have each patient in the flattest position as possible to keep the sacrum at a near-level position in both the anterior/posterior
(AP) and lateral planes. The patient was prepared in the fashion of choice (as preferred by the surgeon) and draped to expose the back from the level of the T12 vertebra to just below the anus for optimal monitoring of motor response during testing. $\mathrm{C}$-arm fluoroscopy was used in every procedure.

The bilateral S3 nerve root foramen was marked on the patient's skin with a marking pen to denote the midline of the sacrum and a horizontal line to denote the level of the sacroiliac joints for reference points. This was followed by marking the medial edge of the bilateral S3 foramina and the midline of the visualized S3 foramina bilaterally to denote the most lateral aspect for which the needle/lead should be placed in the foramina from an AP radiologic perspective. The landmarks of the sacrum in the AP and lateral views (respectively) that should be identified for the procedure are denoted (Fig. 1A, B). Once the bilateral S3 nerve root foramina were delineated, the spinal needles were placed to localize the S3 nerve. Once all sacral landmarks were identified, the needle(s) were positioned, tested, and repositioned until optimal placement was achieved in both the AP and lateral views (Fig. 1C, D), respectively.

\section{RESULTS}

The following figures are a representation of "improper" needle placement and subsequent lead placement, and the reproducible motor and sensory responses that can be expected with common malpositioning as observed over the course of $400+$ implants. It should be noted that each figure represents gross needle position and depth (placed approximately $1 \mathrm{~cm}$ past the anterior edge of the sacrum for each figure). Additionally, the differential change needed to achieve optimal needle placement often only requires diminutive adjustments of the needle (versus gross or exaggerated movements) to ultimately deliver optimal needle/lead position (optimal placement as defined by the current International Continence Society guidelines for optimal placement).

AP and lateral views that will yield an S2 and S4 response when placing the needle during SNM procedures are demonstrated (Figs. 2-5). When conceptualizing in a 3-dimensional aspect, results of these types of needle placements yield far different responses than what is considered optimal.

\section{Predicted Response (Fig. 2)}

- Anal Bellows: Atypical bellowing. Not a traditional inward pulling of the upper gluteal fold, but rather a flexing of the gluteal muscle (specific to the correlating body side of needle 

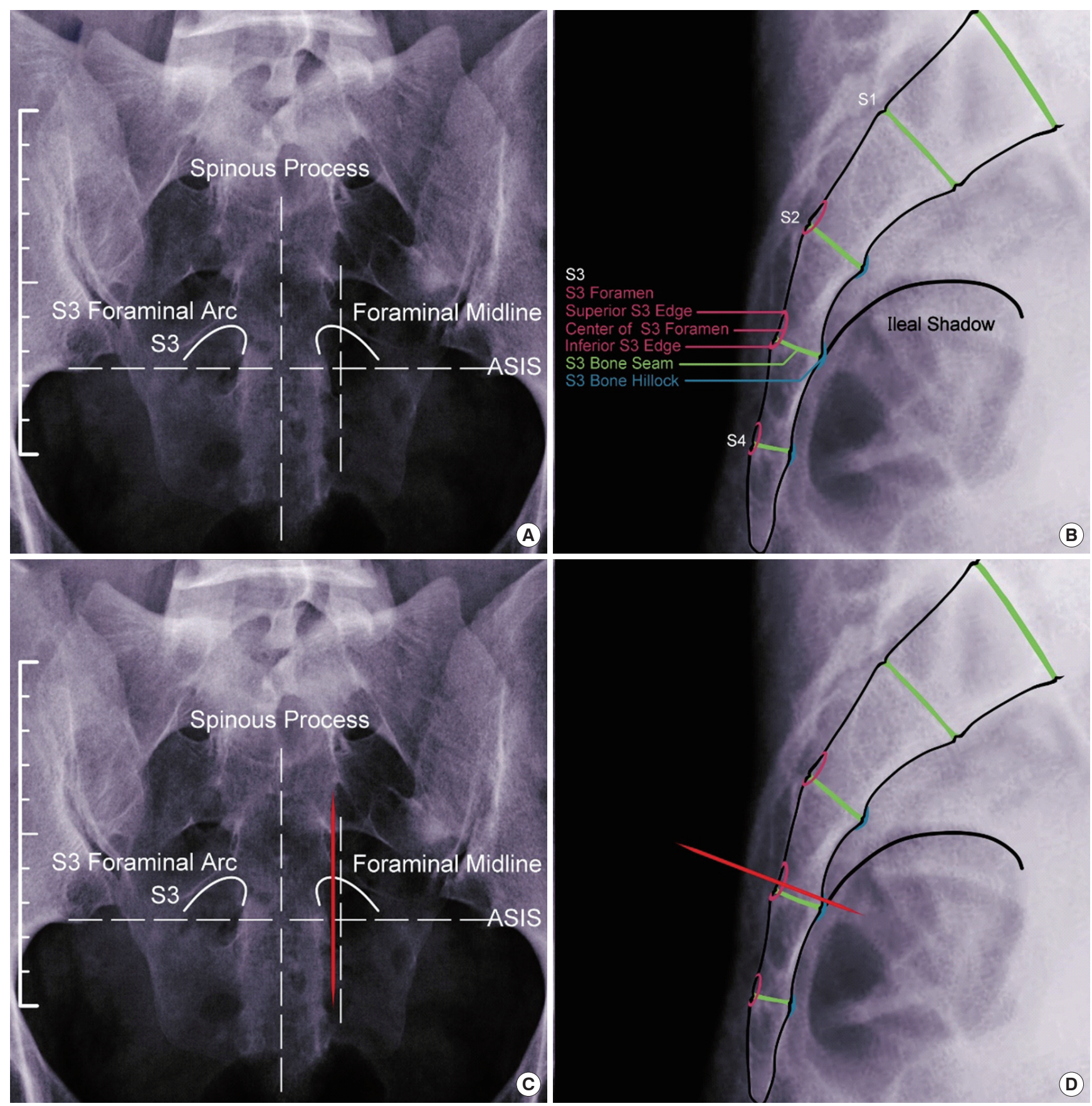

Fig. 1. Anteroposterior (A) and lateral (B) sacral landmarks with optimal needle placement, anteroposterior (C), and lateral (D). ASIS, anterior superior iliac spine.

placement) and concurrent clenching of the anal sphincter.

- Toes: Atypical toe(s) flexion. There are 2 plausible motor responses (dependent on which section of the nerve fiber is being stimulated):

(1) Dramatic plantar flexion of the great toe or full plantar flex- ion of the toe(s), with concurrent quivering of the toe(s) that starts in the arch of the foot and extends to the toe(s).

(2) Dramatic outward rotation of the heel (Specific to the correlating body side of needle placement), and concurrent flexing of the calf muscle. 

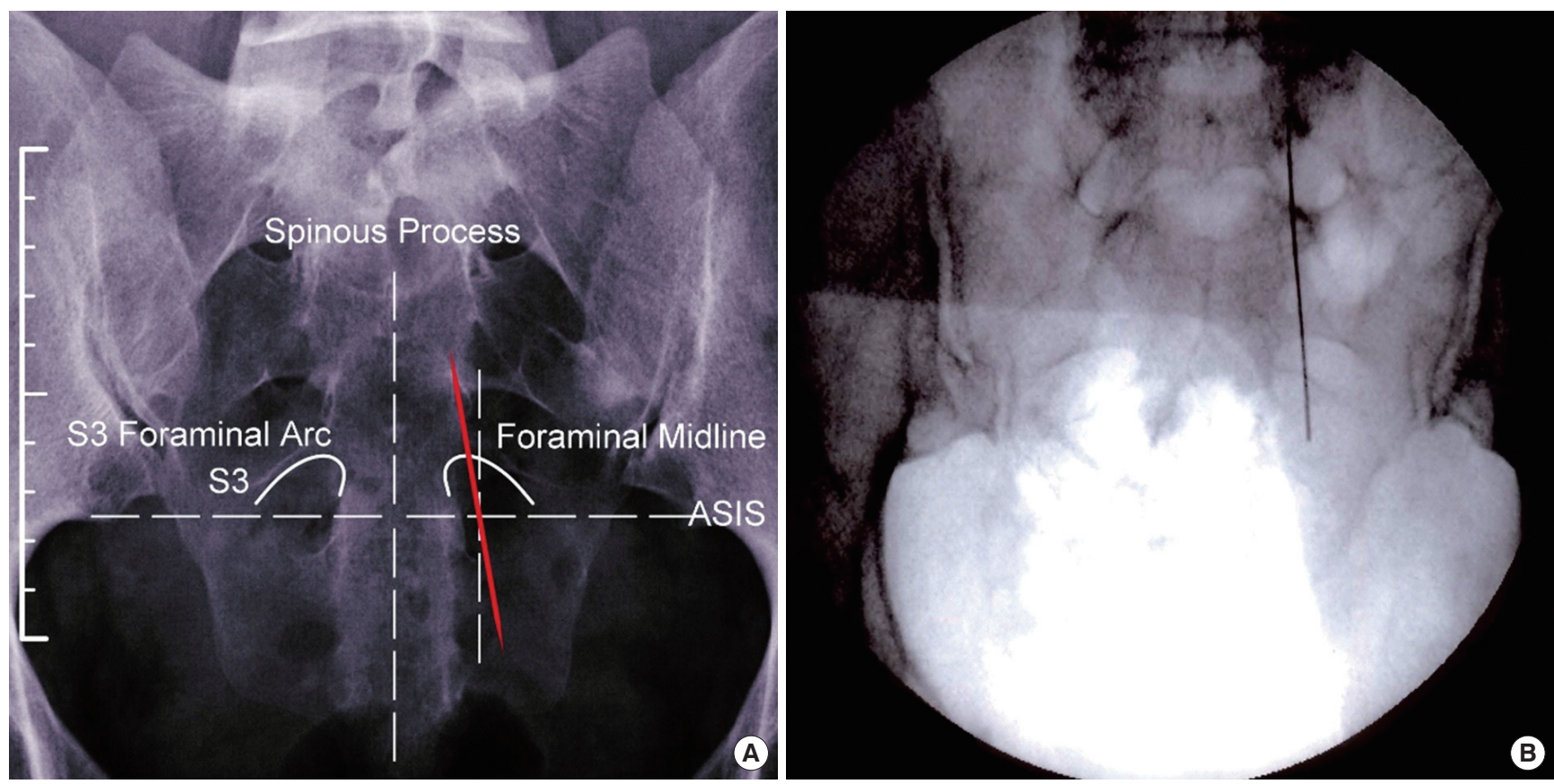

Fig. 2. Anteroposterior view of needle crossing midline of foramen yielding S2 response (medial to lateral needle trajectory). ASIS, anterior superior iliac spine.
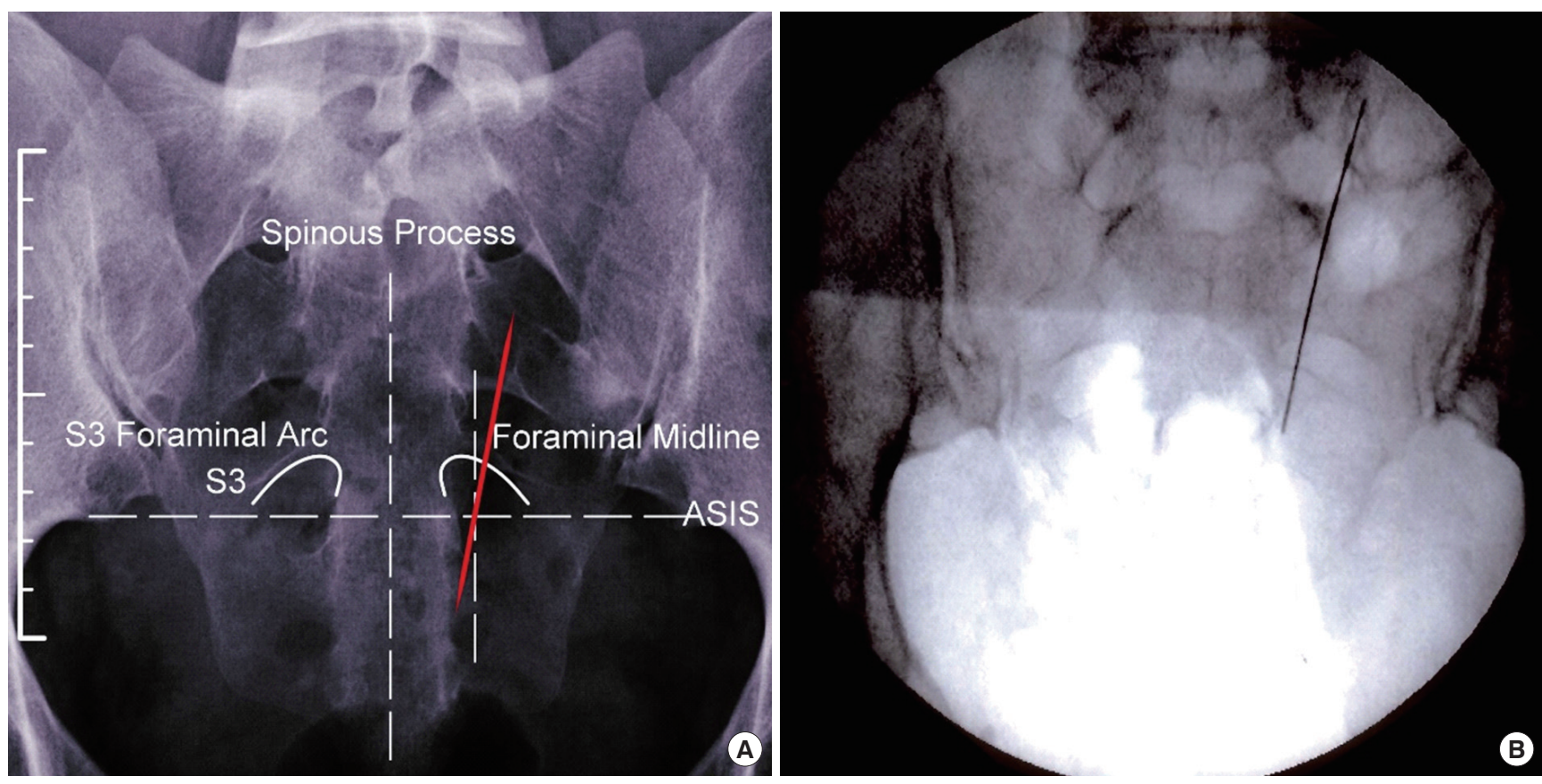

Fig. 3. Anteroposterior view of needle crossing midline of foramen yielding $\$ 4$ response or no response (lateral to medial needle trajectory).

\section{Reason for Predicted Response (Fig. 2)}

- Even though the needle is in the $\mathrm{S} 3$ foramen, the extreme medial to lateral trajectory of the needle (viewed on the image from superior to inferior) is affecting the significantly distal section of the S2 nerve fiber. One will observe 1 of the 2 responses specific to the location of the stimulation on this sec- 

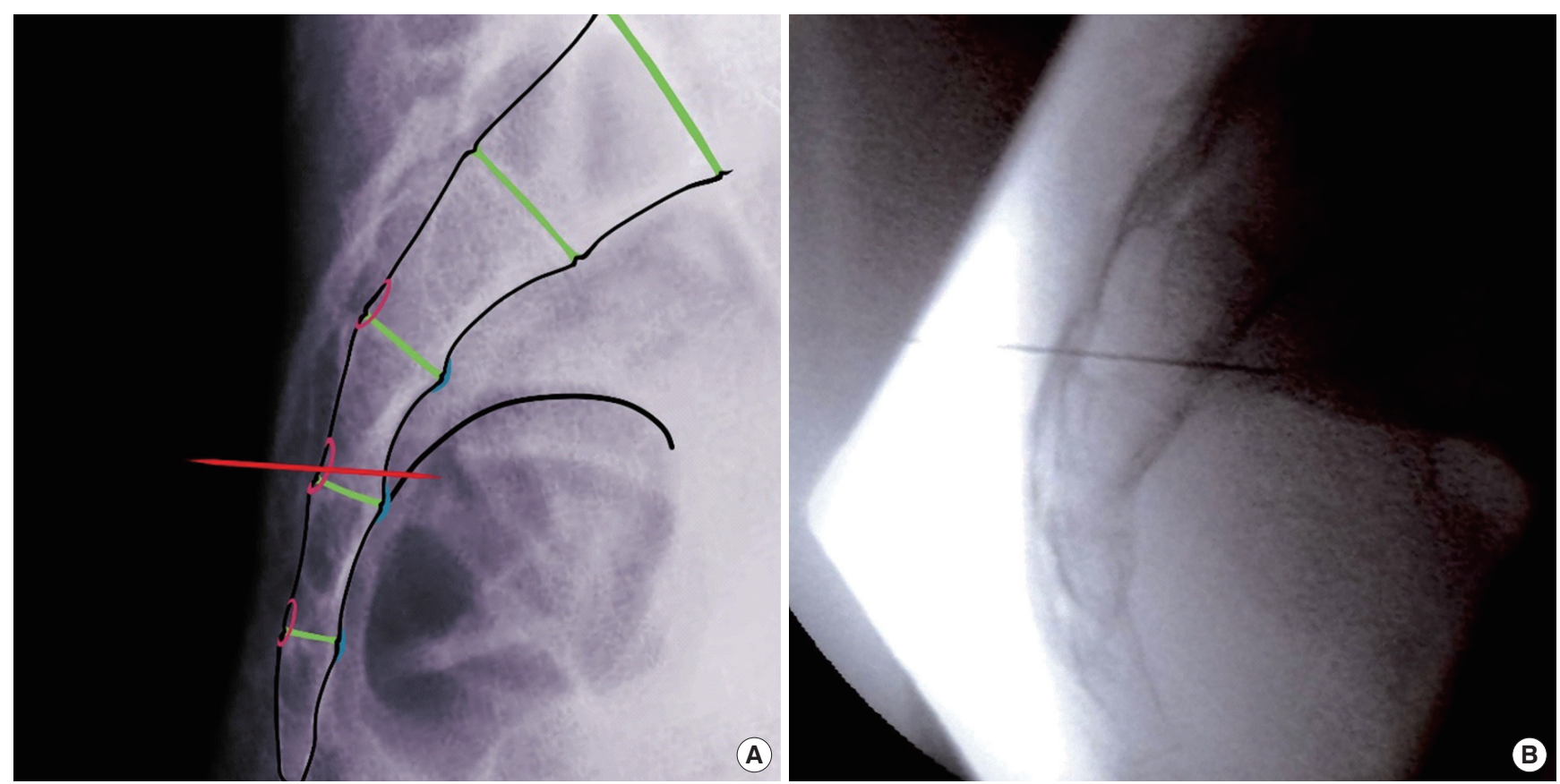

Fig. 4. Lateral view of needle crossing the width of foramen yielding S2 response (inferior to superior needle trajectory).
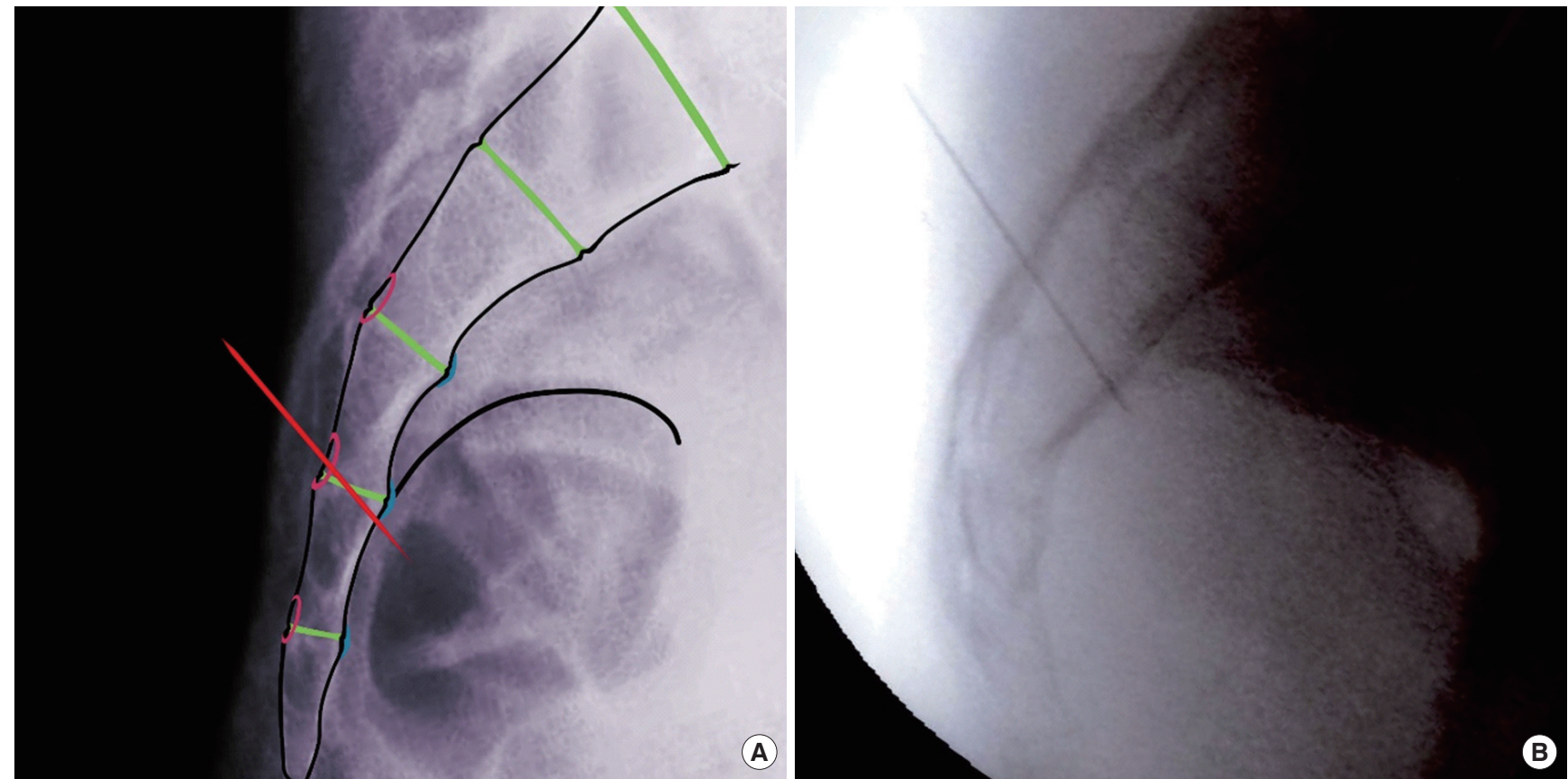

Fig. 5. Lateral view of needle crossing the width of foramen yielding S4 response (superior to inferior needle trajectory).

tion of the nerve fiber.

\section{Predicted Response (Fig. 3)}

- Anal Bellows: Atypical bellowing. Not a traditional inward pulling of the upper gluteal fold, but rather a strong inward pulling of the gluteal cleft. Additionally, it can be observed that no muscular responses occur. It should be noted that no observed response will only occur if the needle is stimulating the 
proximal section of the $S 4$ nerve root. If the needle bypasses the nerve root completely, then no muscular responses will be observed. This response is usually predicated by the depth of placement of the foraminal needle into the sacrum.

- Toes: There will be no response observed regardless of the depth of needle placement.

\section{Reason for Predicted Response (Fig. 3)}

- Even though the needle is placed in the S3 foramen, the extreme lateral to medial trajectory of the needle (viewed on the image from superior to inferior) is either stimulating the proximal section of the S4 nerve fiber and will yield exaggerated S4 muscular motor responses or the needle is in a "dead space" of the sacrum. This will result in a bypassing of all nerves and will not yield any muscular motor responses. Again, this is dependent on the depth of which the needle is placed into the sacrum.

\section{Predicted Response (Fig. 4)}

- Anal Bellows: Atypical bellowing. This is not a traditional inward pulling of the upper gluteal fold, but rather a flexing of the gluteal muscle (specific to the correlating body side of needle placement) and concurrent clenching of the anal sphincter.

- Toes: Atypical toe(s) flexion. There are 2 plausible responses (dependent on which section of the nerve fiber is being stimulated):

(1) Dramatic plantar flexion of the great toe or full plantar flexion of the toe(s), with concurrent quivering that begins in the arch of the foot and extends to the toe(s).

(2) Dramatic outward rotation of the heel (specific to the correlating body side of needle placement), and concurrent flexing of the calf muscle.

\section{Reason for Predicted Response (Fig. 4)}

- Even though the needle is placed in the S3 foramen, the extreme inferior to superior trajectory of the needle (viewed on the image from superior to inferior) is stimulating the medial section of the S2 nerve fiber. One will observe 1 of the 2 aforementioned responses specific to the location of stimulation on this section of nerve fiber.

\section{Predicted Response (Fig. 5)}

- Anal Bellows: Atypical bellowing. This is not a traditional inward pulling of the upper gluteal fold, but rather a strong inward pulling of the gluteal cleft. Additionally, it can be observed that no muscular responses occur. It should be noted that no observed response will only occur if the needle is stimulating the medial section of the S4 nerve root. If the needle bypasses the nerve root completely, then no muscular responses will be observed. This response is usually predicated by the depth of the placement of the foraminal needle into the sacrum.

- Toes: There will be no response observed regardless of the depth of needle placement.

\section{Reason for Predicted Response (Fig. 5)}

- Even though the needle is in the S3 foramen, the extreme superior to inferior trajectory of the needle (viewed on the image from superior to inferior) is, either stimulating the medial section of the S4 nerve fiber and will yield exaggerated S4 muscular motor responses or it is in a "dead space" of the sacrum. This will result in a bypassing of all nerves and will not yield any muscular motor responses. Again, this is dependent on the depth of which the needle is placed into the sacrum.

\section{DISCUSSION}

The goal of this paper is to demonstrate that there is a way to place a needle/lead during an SNM procedure that will predictably reproduce a motor and sensory response that is consistently achieved with suboptimal needle angle and subsequent suboptimal lead trajectory. By using the presented figures for predicting these motor and sensory responses, we hope to provide an avenue for practitioners to maximize and, more consistently, identify suboptimal needle/lead placement that will ultimately result in more consistent optimal lead placements during SNM procedures. By recognizing and correcting these common needle trajectory placements and subsequent lead placements intraoperatively, practitioners can more consistently achieve the desired motor and sensory responses with optimal placement. By changing needle trajectory intraoperatively to position the needle approximately $1 \mathrm{~cm}$ superiorly and parallel to the bone seam, the practitioner can achieve optimal placement as set forth by the already existing standardized criteria and as demonstrated by Vaganee et al. [2] showing statistically significant higher mean electromyographic values for leads placed cranially and medially within the $\mathrm{S} 3$ foramen.

Of note, this paper has attempted to define a way to predict motor and sensory responses from SNM therapy in the average patient population. While most patients have a typical anatomical nerve trajectory, we acknowledge that there is a small per- 
centage of the patient population who have nerve trajectory anomalies, and this is oftentimes not recognized until needle/ lead placement is attempted during one of these SNM procedures. This is in addition to those patients who may have a nerve trajectory that is a bit more medial, lateral, anterior, or posterior than is typical. Again, this is oftentimes not recognized until stimulation response is tested during the procedure. It is the goal of this manuscript to provide a "guide" to allow practitioners to ultimately be comfortable enough to predict motor and sensory responses in the event of placing a needle/ lead in a patient who has a nerve trajectory that is atypical and recognizing the improper trajectory of the lead on sacral imaging and patient presentation to the office if suboptimal results are experienced by the patient. This is supported by Deng et al. [3], who surmised that more judicious use of sacral X-ray was needed to identify suboptimal lead placement or migration when a patient is receiving suboptimal response from an existing lead. Additionally, Hijaz et al. [4], proposed a postimplant algorithm and better understanding of impedance readings to troubleshoot suboptimal outcomes as a means of maximizing optimal patient experience with SNM. By considering the 3-dimensional spatial trajectory of the S3 nerve, and following this presented guide, practitioners can achieve the most optimal lead placement with consistently reproducible outcomes that include S3 motor and sensory response on all 4 leads at $\leq 2 \mathrm{~V}$. This will potentially afford each patient the best possible outcome by ensuring every lead contact point is in optimal contact with the S3 nerve, in the most efficient way possible. Additionally, by immediately recognizing suboptimal positioning of the needle intraoperatively and correcting the needle trajectory as well as recognizing suboptimal lead placement on imaging studies of patients presenting to the office with complaints of poor symptom control, practitioners can potentially ensure that the best possible outcome can be obtained, and the longevity of the device will be maximized. Thus, potential for increasing the likelihood for greater patient satisfaction can be achieved. This conclusion was also demonstrated and summarized by Matzel et al. [5], when publishing their findings demonstrating optimal needle/lead placement techniques. This paper conversely demonstrates an adjunct for needle/lead placement to bring implantation technique full circle for practitioners.

While studies are now investigating the use of ultrasound to place the SNM needles to reduce radiation exposure as were conducted by Shakuri-Rad et al. [6], this guide uses fluoroscopy exclusively. It is our hope that once the experienced implanter masters optimal needle placement with the fluoroscopic guidance and recognizes suboptimal needle placement intraoperatively as demonstrated in this guide, other avenues for easier and more efficient needle/lead placement with ultrasound guidance can be utilized. This would not only eliminate radiation exposure for the patient, but it would also potentially allow practitioners to perform the trial and implant in the office/ambulatory surgery center setting with more efficiency and less reliance on support staff. This ease of use is especially important to consider when recent studies have indicated that approximately $15 \%$ of patients progress to a third-line therapy with FPMRS-trained physicians while only approximately $1 \%$ of patients are treated with third-line therapies by a generalist [7]. Ostensibly, if ease of use can be improved upon with improved recognition of suboptimal needle placement intraoperatively and increased recognition with the associated motor and sensory responses, then it is our hope that this will lower consensus reservations to treat patients with "procedurally difficult" or prospectively more involved treatment options by facilitating more optimal needle placement. In turn, this will allow more patients who are currently suffering to not only receive beneficial treatment, but to also potentially receive a much higher degree of successful outcome.

\section{ACKNOWLEDGMENTS}

The authors wish to thank Mr. Jeremy Fetter for his graphic design talents helping to produce the figures for this manuscript.

\section{AUTHOR CONTRIBUTION STATEMENT}

- Full access to all the data in the study and takes responsibility for the integrity of the data and the accuracy of the data analysis: $M C B, C C$

- Study concept and design: $M C B, C C$

- Acquisition of data: $M C B, C C$

- Analysis and interpretation of data: $M C B, C C$

- Drafting of the manuscript: $M C B, C C$

- Critical revision of the manuscript for important intellectual content: $M C B, C C$

- Statistical analysis: $M C B, C C$

- Administrative, technical, or material support: $M C B$

- Study supervision: $M C B$ 


\section{REFERENCES}

1. Goldman HB, Lloyd JC, Noblett KL, Carey MP, Castaño Botero JC, Gajewski JB, et al. International Continence Society best practice statement for use of sacral neuromodulation. Neurourol Urodyn 2018;37:1823-48.

2. Vaganée D, Voorham J, Voorham-van der Zalm P, De Wachter S. Needle placement and position of electrical stimulation inside sacral foramen determines pelvic floor electromyographic response-implications for sacral neuromodulation. Neuromodulation 2019;22:709-15.

3. Deng DY, Gulati M, Rutman M, Raz S, Rodríguez LV. Failure of sacral nerve stimulation due to migration of tined lead. J Urol 2006;175:2182-5.
4. Hijaz A, Vasavada SP, Daneshgari F, Frinjari H, Goldman H, Rackley R. Complications and troubleshooting of two-stage sacral neuromodulation therapy: a single-institution experience. Urology 2006;68:533-7.

5. Matzel KE, Chartier-Kastler E, Knowles CH, Lehur PA, MuñozDuyos A, Ratto C, et al. Sacral neuromodulation: standardized electrode placement technique. Neuromodulation 2017;20:816-24.

6. Shakuri-Rad J, Cicic A, Thompson J. Prospective randomized study evaluating ultrasound versus fluoroscopy guided sacral InterStim ${ }^{\circledR}$ lead placement: a pilot study. Neurourol Urodyn 2018;37: 1737-43.

7. Schieszer J. Use of third-line OAB treatments examined. Urol Times 2018;46:14. 